\title{
EMPLOI DU FLUOTHANE EN ANESTHÉSIE DENTAIRE
}

\author{
Luc Perreault, M.D.*
}

L'utiLisation du Fluothane dans le cabinet du chirurgien-dentiste s'avère de plus en plus une amélioration sur les techniques employées antérieurement. Alors qu'avec les techniques précédentes, le chirurgien se limitait la plupart du temps à des procédures aussi brèves que possible et travaillait à la hâte pour abréger la durée de l'anesthésie, il peut maintenant envisager des procédures beaucoup plus longues sans craindre le réveil trop hâtif du patient ou l'hypoxie prolongée que causent de trop fortes concentrations de protoxide d'azote.

La présente étude porte sur une série de 300 cas d'anesthésie au bureau du dentiste: 20 pour cent furent des adultes et 80 pour cent des enfants. Pour les adultes, l'âge variait de 15 à 60 ans alors que l'anesthésie fut adminístrée à des enfants de 2 à 15 ans, la moyenne se situant entre 4 et 10 ans.

En général, seuls les sujets correspondant aux risques I et II de la classification Saklad-Meyer furent acceptés: La durée de l'intervention variait de cinq minutes à 1.30 heure avec une moyenne de 30 minutes. Le genre d'intervention s'est limité la plupart du temps aux extractions et aux obturations dans un rapport de 30 pour cent d'extractions pour 70 pour cent d'obturations. Les cas d'obturations se sont rencontrés à peu près tous chez les enfants. Il y eut trois cas de chirurgie chez l'adulte dont deux cas de dents incluses et un cas d'alvéolectomie.

La préparation des sujets est la plus simple possible. ${ }^{2}$ Le jeûne de quatre à cinq heures est exigé. On ne donne pas de prémédication, quoique il nous est arrivé au début de la série de donner aux enfants nerveux ${ }_{1 / 2}^{\prime 2}$ grain de nembutal. Par la suite, nous avons préféré la préparation psychologique par le dentiste au cours de la ou des visites antérieures.

L'appareillage employé est aussi simple. Un appareil Heidbrink portatif (Fig. 1), un vaporisateur à Trilene fixé à la sortie des gaz, un inhalateur nasal no 6 (Ohio) (Fig. 2) constituent l'essentiel de l'instrumentation. Quelquefois, chez les jeunes enfants, nous avons employé la valve MIE avec un petit masque malléable (Fig. 3) qui s'adapte bien à la respiration nasale. L'intubation oro-trachéale fut employée dans un cas de chirurgie, mais ne s'est pas avérée supérieure à la technique du nasque nasal.

L'induction se fait avec le patient en position assise. Chez l'enfant, elle est conduite assez rapidement avec le mélange $\mathrm{N}_{2} \mathrm{O} / \mathrm{O}_{2}$ en proportions égales auquel on ajoute rapidement des concentrations crosssantes de Fluothane. La même technique est employée chez la plupart des adultes. Dans certains cas, par exemple les hommes costauds ou certaines femmes qui le désirent absolument, nous employons du penthotal pour amorcer l'induction, les doses données variant entre $150 \mathrm{mg}$. et $300 \mathrm{mg}$. Lorsque nous employons le penthotal, nous donnons

"Hôpital St-Joseph de Rosemont, Montreal 36, P.Q.

Can. Anaes. Soc. J., vol. 8, no. 5, September, 1961. 


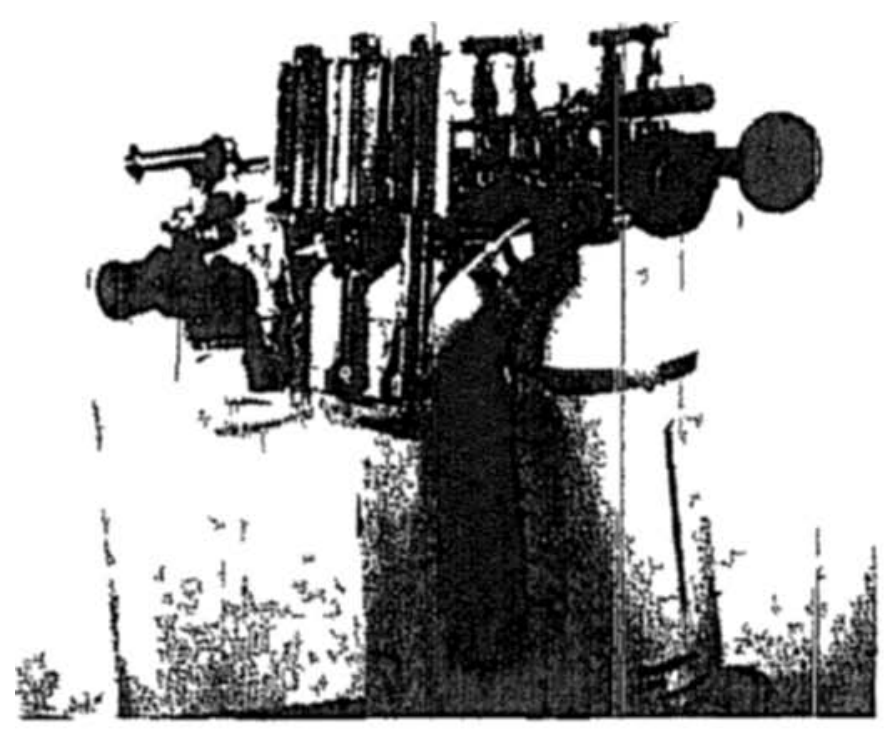

Figure 1

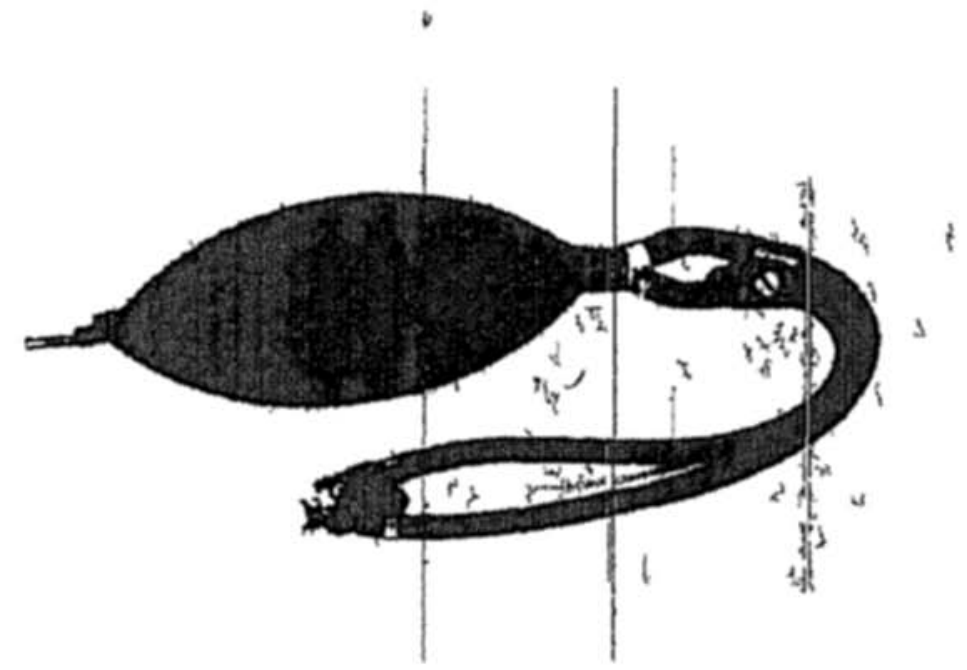

FiGURE 2

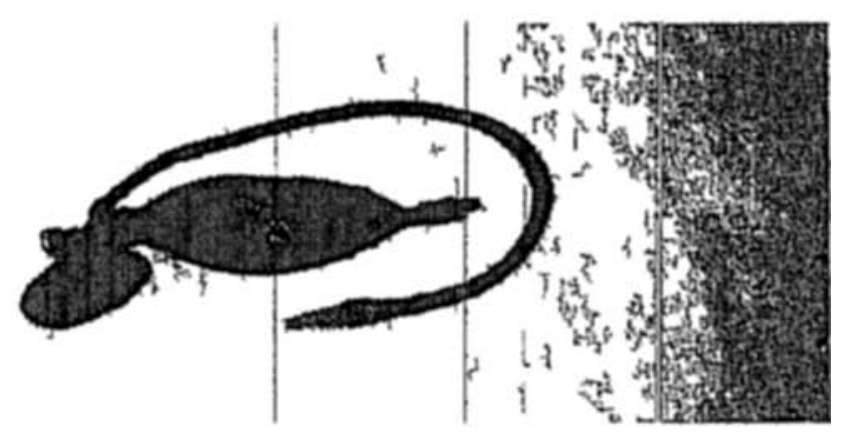

FIC URL 3

toujouis 1 plus petıte dos susceptıble de provoquicı le sommeıl de taçon a eviter une predomm ance ragale qui favoriser ut l hypotension

Li muntenance se fait avec Pıotoxide Fluothane $O$ Le débit total variant entre 8 a $10 \mathrm{~L}$ et quelquefors davantage surtout au cous des procedures pro longees ou 1 on $s$ eftorce de donner un debit/minute supeneur au volume/minute du patient de façon a prevenı au maxımum la ıeınspiration qui favorıseraıt une 
rétention de $\mathrm{CO}_{2}$ et qui modifierait les concentrations de Fluothane dans l'atmosphère inspiré.

Lorsque le patient a atteint le degré d'anesthésie désiré, on place l'ouvre-bouche et un barrage pharyngé bien étanche. Ce barrage prévient la respiration orale et empêche les corps étrangers (sang, amalgame, etc.) de descendre dans le larynx et de là dans les bronches.

L'ouverture du vaporisateur varie avec le débit des gaz et la température du liquide, mais dans la moyenne des cas, une ouverture aux $\% 3$ procure une anesthésie juste assez profonde pour abolir la réaction à la douleur. La position centrée des globes oculaires, le degré de dilatation pupillaire, la vitesse du pouls et l'absence de signes témoignant d'une réaction à la douleur sont les principaux critères sur lesquels on se base pour juger de la profondeur de l'anesthésie. En aucun temps, on tolère une mydriase fixe que l'on considère comme un signe d'une anesthésie trop profonde. L'amplitude respiratoire est bien surveillée de même que la perméabilité du nasopharynx. Dans les cas où l'on note une résistance à la respiration, une luxation antérieure du maxillaire inférieur fait disparaître cette résistance.

Les avantages de cette technique sont nombreux. Pour le patient, l'induction est agréable, tant pour l'adulte que pour l'enfant. Le réveil est rapide et après quelques minutes, l'élimination est à peu près complète, de sorte que le patient se sent très bien. L'absence de nausées et de vomissements est remarquable tant dans la période du réveil que dans les heures qui suivent lanesthésie. Aussi 95 pour cent des sujets peuvent commencer à s'alimenter environ deux heures après la fin de l'anesthésie. L'absence d'hypoxie au cours de l'anesthésie contribue pour beaucoup à la sécurité de la technique et au bien-être du patient au réveil. La pression artérielle que l'on peut apprécier par la qualité du pouis ne subit jamais de chute importante, et la bradycardie n'est jamais marquée.

Du côté de l'opérateur, les avantages ne sont pas moindres. Les cauchemars et l'agitation de la période d'induction'si souvent observée avec le protoxide d'azote sont absents avec le Fluothane. La puissance du Fluothane permet d'obtenir un plan d'anesthésie suffisant pour éliminer la réponse åux stimuli douloureux et par conséquent procure une tranquillité absolue pour l'opérateur. En même temps, le relâchement du maxillaire et du pharynx est complet et permet de placer avec facilité louvre-bouche et le tampon pharyngé. L'absence de salive et la sécheresse complète des muqueuses est un autre avantage très important qui procure des conditions idéales pour les obturations. De tous les cas d'obturations pratiquées selon cette technique, seulement deux enfants ont développé des abcès sur une dent réparée, et dans les deux cas, il s'agissait de caries très profondes où le dentiste a tenté une obturation mais en évaluant d'avance les chances de succès comme minimes. Enfin, le Fluothane a comme dernier avantage d'être un des seuls anesthésiques à allier en même temps la puissance et la non-explosivité.

Comme désavantage, il faut noter le danger de surdosage. Le produit a le défaut de sa principale qualité: en effet, sa puissance est telle, que le dosage doit ètre constamment surveillé et les signes d'anesthésie notés avec attenlion. Le coût du produit est un autre facteur à considérer, mais les quantités nécessaires pour une anesthésie dentaire sont assez minimes de sorte que le prix de revient 
quoique supérieur au protoxide seul ou associé au pentothal, démeure encore dans des limites convenables.

Au cours de ces 300 cas, aucun accident n'est survenu chez les patients anesthésiés avec du Fluothane. Seul un cas de lipothimie avec pâleur des téguments et transpiration fut noté: il s'agissait d'une jeune fille de 18 ans qui, à notre insu, n'était pas à jeun depuis le temps requis. Le vomissement corrigea cet état d'hypervagotonie.

\section{RÉSUMÉ et ConClusion}

Il s'agit d'une observation portant sur 300 cas d'anesthésie au protoxydeFluothane- $\mathrm{O}_{2}$ chez le dentiste. Quatre-vingt pour cent des cas étaient des enfants entre quatre et dix ans pour la plupart. La technique s'est avérée avantageuse au point de vue sécurité pour le patient, et facilité de travail pour l'opérateur. Elle offre l'avantage de pouvoir pratiquer des obturations dans des conditions idéales chez des enfants qui, autrement, auraient dû subir des extractions dentaires.

Le Fluothane employé en anesthésie dentaire s'est révélé une technique sûre et efficace, et apporte une amélioration sensible, surtout en ce qui regarde la conservation des dents chez les enfants.

\section{SUMMARY}

This is an observation of 300 patients who underwent general anesthesia with Nitrous-Oxide-Fluothane- $\mathrm{O}_{2}$ for dental treatments of some kind or other. Eighty per cent of those patients were children whose age ranged from 2 and 15 years, with an average age of 4-10 years.

The technique used has proven useful because it is safe and permits the dentist to work more freely. Fluothane has the advantage of producing dryness of the mouth and so gives ideal conditions for filling teeth which would otherwise be extracted. 